\title{
Mirrors, mirrors, everywhere?
}

The discussion about a possible role of mirror neurons in learning and social cognition has been hampered by the lack of direct evidence that such neurons exist in humans. Fried and colleagues have now recorded single-cell activity from neurons in several brain areas in humans and report that some of these neurons show mirror-like activity.

The authors recorded neuronal activity in patients with implanted electrodes while the patients observed or executed grasping hand

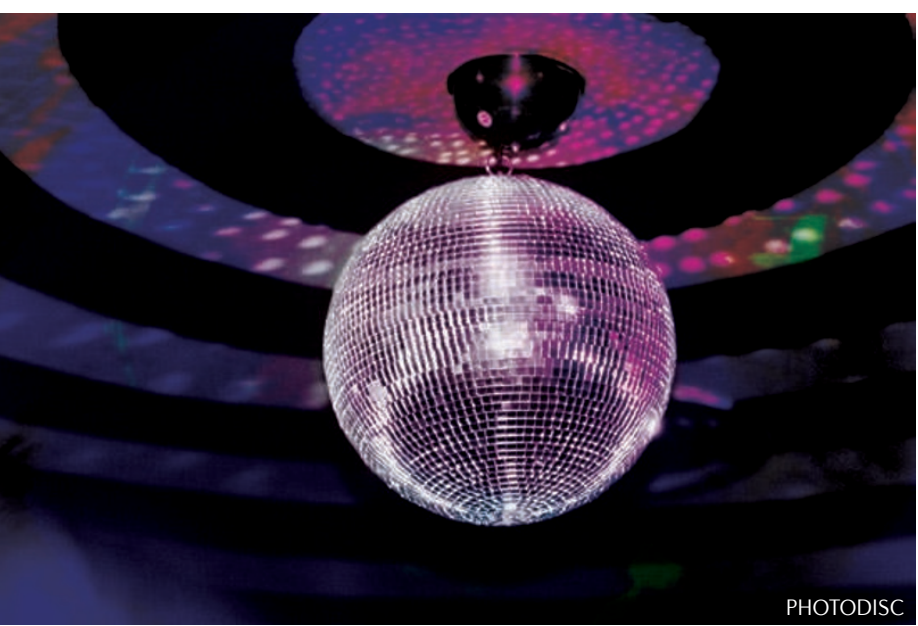

actions and facial expressions. They recorded from neurons in the medial frontal cortex (supplementary motor area and anterior cingulate cortex) and the medial temporal cortex (entorhinal cortex, hippocampus, parahippocampal gyrus and amygdala). In the hippocampus, the parahippocampal gyrus, the entorhinal cortex and the supplementary motor area, a higher percentage of the recorded neurons than expected by chance changed their activity when the participant observed a particular action and when he or she executed that action.

Of the 68 'matching cells' detected, 33 showed increased firing during both the observation and execution of a particular action and 21 cells showed decreased firing in both conditions. Interestingly, in the remaining 14 neurons, the firing rate increased in one condition but decreased in the other.

In monkeys, mirror neurons have been recorded in the lateral aspects of the frontal and parietal cortex. As the electrodes in the present study were not placed in the regions homologous to these areas, this study could not establish whether they also contain mirror neurons in humans. The perhaps surprising finding of mirrorlike neurons in the medial temporal lobe led the authors to suggest that activity in these cells might represent the reactivation of a memory trace for the observed or executed action. They also speculated that the neurons showing opposite responses to action observation and execution might have a role in suppressing the execution of observed actions to ensure that we do not imitate every action we see.

Arguably, neurons that decrease their firing rate during action observation cannot be considered 'classical' mirror neurons, and these findings will no doubt fuel the debate about the definition and functional importance of mirror neurons.

Leonie Welberg

ORIGINAL RESEARCH PAPER Mukamel, R. et al. Single-neuron responses in humans during execution and observation of actions. Curr. Biol. 20, 750-756 (2010)

FURTHER READING Rizzolatti, G. \& Sinigaglia, C. The functional role of the parieto-frontal mirror circuit: interpretations and misinterpretations. Nature Rev. Neurosci. 11, 264-274 (2010) 